\title{
Comparison of dispersion-corrected exchange-correlation functionals using atomic orbitals
}

\author{
Aleksandr V. Terentjev $\odot,{ }^{1}$ Lucian A. Constantin $\odot,{ }^{2}$ Emilio Artacho $\odot,{ }^{3,4,5}$ and J. M. Pitarke $\odot^{4,6}$ \\ ${ }^{1}$ Donostia International Physics Center (DIPC), E-20018 Donostia, Basque Country, Spain \\ ${ }^{2}$ Center for Biomolecular Nanotechnologies @ UNILE, Istituto Italiano di Tecnologia, Via Barsanti, I-73010 Arnesano, Italy \\ ${ }^{3}$ Theory of Condensed Matter, Cavendish Laboratory, University of Cambridge, J. J. Thomson Avenue, Cambridge CB3 OHE, United Kingdom \\ ${ }^{4}$ CIC nanoGUNE and DIPC, Tolosa Hiribidea 76, E-20018 Donostia, Basque Country, Spain \\ ${ }^{5}$ Ikerbasque, Basque Foundation for Science, E-48013 Bilbo, Basque Country, Spain \\ ${ }^{6}$ Materia Kondentsatuaren Fisika Saila and Centro Física Materiales CSIC-UPV/EHU, \\ 644 Posta kutxatila, E-48080 Bilbo, Basque Country, Spain
}

(Received 8 October 2019; published 20 December 2019)

\begin{abstract}
We assess several recently developed dispersion-corrected exchange-correlation functionals [PBE + VV10L of H. Peng and J. P. Perdew, Phys. Rev. B 95, 081105 (2017); SG4 + VV10m of A. V. Terentjev et al., Computation 6, 7 (2018); and PBEsol+VV10s of A. V. Terentjev et al., Phys. Rev. B 98, 214108 (2018)] for the adsorption of a noble-gas atom/monolayer as well as a graphene on the $\mathrm{Cu}(111), \operatorname{Pt}(111), \operatorname{Pd}(111), \operatorname{and} \mathrm{Ag}(111)$ close-packed surfaces. Most of these systems are characterized by weak interactions between the surface and the atom/monolayer, where the van der Waals interaction dominates. Here we investigate the above mentioned dispersion-corrected exchange-correlation functionals using atomic orbitals instead of plane waves applied in earlier works. We find that PBEsol+VV10s is an optimal functional for such hybrid interfaces, being accurate for both the equilibrium distance and the adsorption energy and providing realistic potential-energy curves. Moreover, we also show that PBEsol+VV10s and SG4+VV10m are both very accurate for the investigation of surface formation energies of all transition metals under consideration.
\end{abstract}

DOI: 10.1103/PhysRevB.100.235439

\section{INTRODUCTION}

In the Kohn-Sham (KS) density functional theory (DFT) $[1,2]$, the exchange-correlation (XC) energy functional,

$$
E_{x c}\left[n_{\uparrow}, n_{\downarrow}\right]=\int d \mathbf{r} n(\mathbf{r}) \epsilon_{x c}(\mathbf{r}),
$$

accounts for all many-body effects beyond the Hartree method and must be approximated. Here $\epsilon_{x c}(\mathbf{r})$ is the XC energy per particle and $n=n_{\uparrow}+n_{\downarrow}$ is the electron density, $n_{\uparrow}$ and $n_{\downarrow}$ representing spin-up and spin-down electron densities, respectively.

Semilocal XC functionals, such as generalized gradient approximations (GGAs) [3] and meta-GGAs [4], are the most used in electronic-structure calculations of solids, because of their accuracy and little computational demand. While GGAs are based on the knowledge of the electron density and the electron-density gradient, meta-GGAs are derived from the knowledge of the KS kinetic-energy density as well.

Semilocal XC functionals, which are all characterized by a localized XC hole density [5,6], can recognize various types of interactions and density regimes, such as covalent, metallic, and some short-ranged weak bonds [4,7-9], can be utilized for an accurate description of surface asymptotics [10], and can describe the dimensional crossover of the XC energy [11-13] for various confinements [14]; but they cannot possibly describe correctly van der Waals (vdW) interactions even at short range [15]. Whenever vdW interactions play a role, one can use the so-called DFT-D semiempirical approach [16-29], which is based on the cancellation of various errors especially at a short range [15]. We recall that DFT-D introduces a force-field correction to the semilocal result, of the form

$$
E_{\mathrm{vdW}}=s_{6} \sum_{\text {pairs }} E_{\mathrm{vdW}}^{\mathrm{LR}}(R) f_{\mathrm{dmp}}(R), \quad E_{\mathrm{vdW}}^{\mathrm{LR}}=-\frac{C_{6}}{R^{6}},
$$

where $R$ is the interatomic distance, $s_{6}$ is a scaling factor dependent on the semilocal functional, $f_{\mathrm{dmp}}(R)$ is a damping function, and $E_{\mathrm{vdW}}^{\mathrm{LR}}$ represents the long-range $\mathrm{vdW}$ interaction with $C_{6}$ being the dispersion coefficient for each atom pair.

Another approach for adding dispersion effects to a semilocal functional is the so-called Rutgers-Chalmers method [30-35], which uses a nonlocal vdW correlation functional of the following form:

$$
E_{c}^{\mathrm{nl}}[n]=\int d \mathbf{r} n(\mathbf{r}) \varepsilon_{c}^{\mathrm{nl}}[n]=\int d \mathbf{r} \int d \mathbf{r}^{\prime} n(\mathbf{r}) \Phi\left(\mathbf{r}, \mathbf{r}^{\prime}\right) n\left(\mathbf{r}^{\prime}\right),
$$

where the kernel $\Phi\left(\mathbf{r}, \mathbf{r}^{\prime}\right)=\Phi\left[n(\mathbf{r}), n\left(\mathbf{r}^{\prime}\right), \nabla n(\mathbf{r}), \nabla n\left(\mathbf{r}^{\prime}\right)\right.$, $\left.\left|\mathbf{r}-\mathbf{r}^{\prime}\right|\right]$ recovers exact conditions that are relevant for the $\mathrm{vdW}$ interaction. A number of $\mathrm{vdW}$ kernels are available in the literature [36-39]; in particular, we mention the VV10 kernel [36] and its revision, suitable for plane-wave codes, named rVV10 [39]. Both VV10 and rVV10 have two adjustable parameters $b$ and $c$ assuring the compatibility between the semilocal XC functional and $E_{c}^{\mathrm{nl}}[n]$. Recently, several variations of these $\mathrm{vdW}$ functionals have been proposed: AM05-VV10sol [40], SCAN+rVV10 [41], PBE+rVV10L [42], SG4+rVV10m [43], C09-vdW-DF [44], vdW-DF-cx [45-48], PBEsol+rVV10s [49], and rev-vdW-DF2 [50,51]. All these variations have been tested for many vdW systems, 
such as layered materials [33,39-42,45,49,51-62], rare-gas (RG) solids [43,49,62,63], and molecular solids [43,62].

We mention that the kernels entering Eq. (3), such as VV10 and rVV10, are usually two-body approximations without multilayer screening effects [64], extremely strong screening in 2D systems $[65,66]$, long-range scaling, and dimensional crossovers [64,67-70]. Even if the long-range vdW is described by a 2-body approximation, the intermediate and short-range dispersions depend on the semilocal functional as well. Therefore, despite the fact that these dispersioncorrected functionals are inferior to the random phase approximation (RPA) [11,71-89], which can naturally account for many-body effects up to infinite order, they are useful and accurate for many applications, including layered materials. On the other hand, RPA can correctly describe the vdW interaction in extreme conditions, such as in quasi-two-dimensional systems, but it is much more expensive. Moreover, the RPA results may be very sensitive to the used orbitals (e.g., LDA or PBE orbitals), and fully self-consistent RPA calculations are usually not accessible for large systems. Finally, we recall that the logic of the vdW kernel $\Phi\left(\mathbf{r}, \mathbf{r}^{\prime}\right)$ has been discussed in Ref. [35] using the plasmon-pole description of the Rapcewicz and Ashcroft picture [90].

In this paper, we use the recently developed dispersion-corrected $\mathrm{XC}$ functionals SG4+rVV10m [43] and PBEsol+rVV10 [49] in the framework of the SIESTA method [91]. These functionals were already realized in the plane-wave based Quantum-Espresso (QE) code [92], combining SG4 and PBEsol semilocal functionals with vdW rVV10 kernels $[43,49]$. In the case of SIESTA, we combine SG4 and PBEsol with VV10 (instead of rVV10), keeping the same dispersion parameters $b$ and $c$. The SIESTA method, which uses localized numerical atomic orbitals (NAOs), has a number of advantages with respect to a plane-wave basis set: SIESTA is typically more efficient, as the number of required basis functions is usually smaller, and SIESTA's localized basis set gives a better description of systems where the orbitals are strictly localized in real space, as in the case of adsorption of atoms/molecules on surfaces and in the case of interfaces with different materials. Here we investigate, in the framework of the SIESTA method, new dispersion-corrected functionals that we call PBEsol+VV10s and SG4+VV10m, and we compare to the results one obtains with other functionals such as PBE+VV10L [42] and vdW-DF-cx [45-48].

The paper is organized as follows: In Sec. II, we present a short overview of XC functionals and, in particular, a comparison between the vdW kernels rVV10 and VV10. In Sec. III, we present the computational details of our calculations. In Sec. IV, we report the results we have obtained for the adsorption of RG atom/monolayer and graphene on transition-metal (111) surfaces and also for (111) surface formation energies of various transition metals. In Sec. V, our conclusions are summarized.

\section{OVERVIEW OF XC FUNCTIONALS}

In Fig. 1, we show a comparison of the exchange enhancement factor $F_{x}=\epsilon_{x}^{\mathrm{GGA}} / \epsilon_{x}^{\mathrm{LDA}}$ of the GGA functionals under consideration: PBE, PBEsol, SG4, and cx13. At small

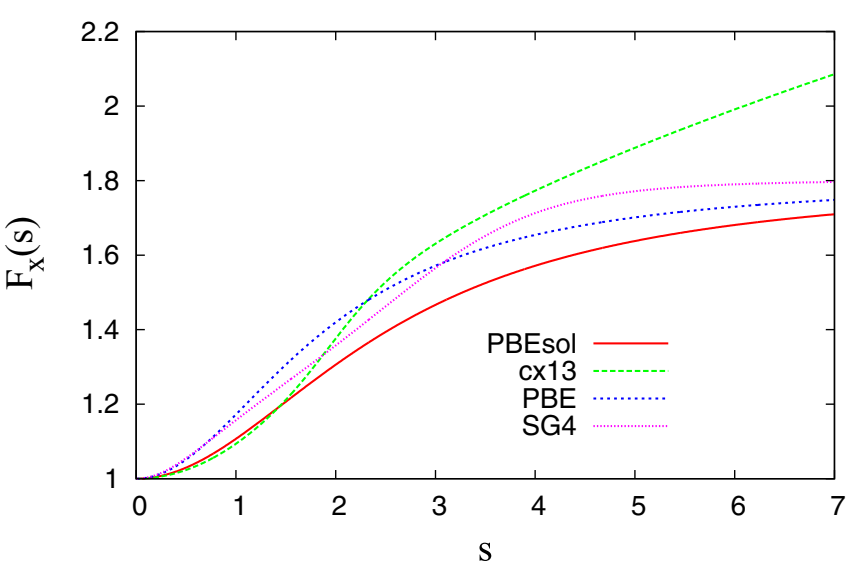

FIG. 1. Exchange enhancement factor $F_{x}$ of the GGA functionals used in this work, versus the reduced gradient $s$.

values of the reduced gradient $s=|\nabla n| /\left[2\left(3 \pi^{2}\right)^{1 / 3} n^{4 / 3}\right]$, the exchange enhancement factor always behaves as $F_{x} \rightarrow 1+$ $\mu s^{2}$, with the functional-dependent coefficient $\mu: \mu^{c x 13}=$ 0.094 is the Vosko-Langreth value [93], $\mu^{\mathrm{PBEsol}}=10 / 81$ is the exact second-order coefficient [94], $\mu^{\mathrm{PBE}}=0.2195$ [95], and $\mu^{\mathrm{SG} 4}=0.26$ as found from a semiclassical atom theory [96-99]. At large values of $s$, PBE, PBEsol, and SG4 all yield exchange enhancement factors that are close to the maximum value 1.804 given by the Lieb-Oxford bound [100,101], while $F_{x}^{c x 13}$ diverges as $s^{2 / 5}$ recovering the PW86r functional [45]. Note that only cx13 has been constructed with the aim of developing a vdW $\mathrm{XC}$ functional.

The VV10 and rVV10 correlation energy per particle has the form

$$
\varepsilon_{c}[n]=\frac{1}{32}\left(\frac{3}{b^{2}}\right)^{3 / 4}+\frac{1}{2} \int d \mathbf{r}^{\prime} \Phi\left(\mathbf{r}, \mathbf{r}^{\prime}\right) n\left(\mathbf{r}^{\prime}\right) .
$$

Here,

$$
\Phi^{V V 10}\left(\mathbf{r}, \mathbf{r}^{\prime}\right)=-\frac{3}{2} \frac{1}{g(\mathbf{r}) g\left(\mathbf{r}^{\prime}\right)\left[g(\mathbf{r})+g\left(\mathbf{r}^{\prime}\right)\right]}
$$

and

$$
\Phi^{r V V 10}\left(\mathbf{r}, \mathbf{r}^{\prime}\right)=-\frac{3}{2} \frac{1}{g(\mathbf{r}) g\left(\mathbf{r}^{\prime}\right)\left[g(\mathbf{r})\left(\frac{K\left(\mathbf{r}^{\prime}\right)}{K(\mathbf{r})}\right)^{1 / 2}+g\left(\mathbf{r}^{\prime}\right)\left(\frac{K(\mathbf{r})}{K\left(\mathbf{r}^{\prime}\right)}\right)^{1 / 2}\right]},
$$

where $g(\mathbf{r})=\omega_{0}(\mathbf{r}) R^{2}+K(\mathbf{r}), \quad \omega_{0}(\mathbf{r})=\sqrt{\omega_{g}^{2}(\mathbf{r})+\frac{\omega_{p}^{2}(\mathbf{r})}{3}}$, $\omega_{p}^{2}(\mathbf{r})=4 \pi n(\mathbf{r}), \omega_{g}^{2}(\mathbf{r})=c \frac{|\nabla n(\mathbf{r})|^{4}}{n^{4}(\mathbf{r})}, K(\mathbf{r})=b \frac{3 \pi}{2}\left[\frac{n}{9 \pi}\right]^{1 / 6}$, and $R=\left|\mathbf{r}-\mathbf{r}^{\prime}\right|$.

By fitting to $54 C_{6}$ coefficients, the parameter $c$ was originally taken to be $c=0.0093[36,102-104]$ in the vdW kernels VV10 and rVV10 and also in the XC functionals PBE+rVV10L [42] and SCAN+rVV10 [41]. For the description of solids, smaller values of $c$ have been considered: $c=$ $10^{-6}$ in AM05-VV10sol [40] and $c=10^{-4}$ in SG4+rVV10m [43]. In the case of PBEsol+rVV10s [49], one takes

$$
c=c_{0}+\frac{c_{1}}{1+c_{2}\left(s-\frac{1}{2}\right)^{2}},
$$




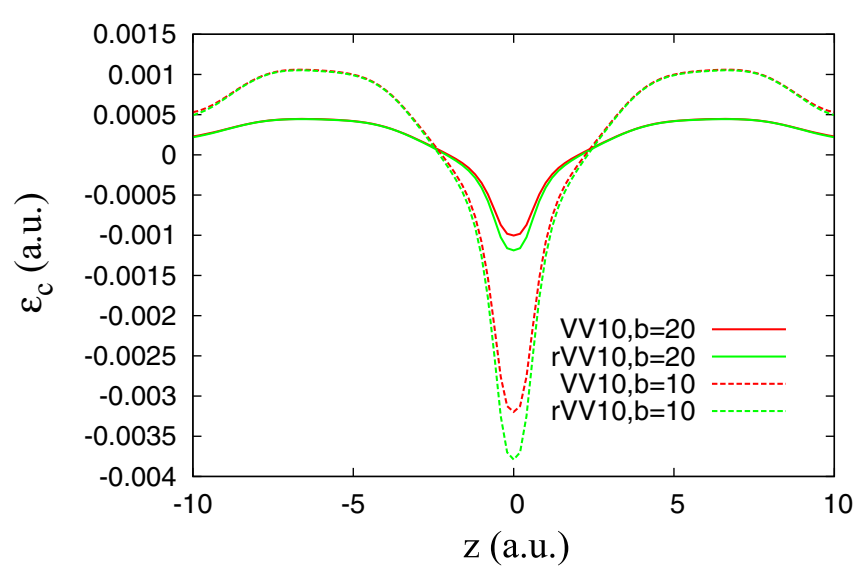

FIG. 2. VV10 and rVV10 correlation energies per particle, versus the distance $z$ perpendicular to two interacting parallel jellium slabs with the electron-density parameter $r_{s}=2.07$. Each slab is $L=5$ a.u. thick. The distance between the slabs is $D=7$ a.u. The parameter $c$ has been taken to be $c=0.0093$ for both VV10 and rVV10. The parameter $b$ has been taken to be $b=10$ and $b=20$.

where $c_{0}=0.0093, c_{1}=0.5, c_{2}=300$, and $s$ is the reduced gradient.

The parameter $b$ is usually fitted to molecules (e.g., the S22 test $[103,104])$ and/or solid systems (e.g., layered materials). This parameter is taken to be $b=5.9$ and $b=6.3$ for the vdW kernels VV10 and rVV10, respectively, $b=10$ for PBE+rVV10L [42] and PBEsol+rVV10s [49], and $b=12$ for SG4+rVV10m [43].

In order to visualize the differences between the VV10 and rVV10 kernels, in Fig. 2 we show the VV10 and rVV10 correlation energies per particle as obtained from Eq. (4) [by using Eq. (5) and Eq. (6), respectively] for two interacting parallel jellium slabs with the electron-density parameter $r_{s}=2.07$. This system was also used in Ref. [49] in order to assess the XC functional PBEsol+rVV10s. We observe that VV10 and rVV10 correlation energies per particle agree very well in most of the space; it is only around $z=0$, in the bonding region, that these position-dependent correlation energies slightly depart from each other. Hence, we conclude that the same values of the parameters $b$ and $c$ can be used for the vdW kernels VV10 and rVV10.

\section{COMPUTATIONAL DETAILS}

The calculations presented here have been done using the SIESTA code [91], hence with a localized NAO basis set and a pseudopotential (PP) representation of core states. The SIESTA pseudopotentials are generated using the program ATOM. We have created scalar relativistic Troullier-Martins pseudopotentials [105] with nonlinear core corrections for all atoms [106]. These pseudopotentials were generated with the following reference configurations: $5 s^{1} 5 p^{0} 4 d^{10} 4 f^{0}$ for $\mathrm{Ag}$, $4 s^{1} 4 p^{0} 3 d^{10} 4 f^{0}$ for $\mathrm{Cu}, 5 s^{1} 5 p^{0} 4 d^{9} 4 f^{0}$ for $\mathrm{Pd}, 6 s^{1} 6 p^{0} 5 d^{9}$ $5 f^{0}$ for $\mathrm{Pt}, 3 s^{2} 3 p^{6} 3 d^{0} 4 f^{0}$ for $\mathrm{Ar}, 5 s^{2} 5 p^{6} 5 d^{0} 4 f^{0}$ for $\mathrm{Xe}$, and $2 s^{2} 2 p^{2} 3 d^{0} 4 f^{0}$ for $\mathrm{C}$. The cutoff radii for the $s, p, d$, and $f$ components of the pseudopotentials are $2.49,2.68$, and 2.49 a.u. for $\mathrm{Ag} ; 2.67,2.57,2.08$, and 2.39 a.u. for $\mathrm{Cu} ; 2.58,2.71$, 2.42, and 2.39 a.u. for Pd; 2.57, 2.70, and 2.57 a.u. for Pt;
$1.58,1.58$, and 1.89 a.u. for Ar; 2.11, 2.11, and 2.58 a.u. for $\mathrm{Xe}$, and 1.54 a.u. for C. Pseudopotentials were generated for PBE, PBEsol, and SG4. The PBE pseudopotential was used for the XC functionals PBE, PBE+VV10L, and vdW-DF-cx; the SG4 pseudopotential was used for the XC functionals SG4 and SG4+VV10m; and the PBEsol pseudopotential was used for the XC functionals PBEsol and PBEsol+VV10s. Pseudopotential transferability was tested by comparing pseudopotential and all-electron calculations. Excitation energies were found to agree within $10 \mathrm{mRy}$.

For all transition metals we use the double- $\zeta s, p$, and $d$ basis; for RG atoms we use the double- $\zeta s$ and $p$ basis with single- $p$ polarization; and for $\mathrm{C}$ we use the double- $\zeta s$ and $p$ basis with double- $p$ polarization. In the case of transition metals and rare gases the basis sets were optimized for dimers with an averaged bond length between the experimental bulk lattice constant and the experimental bond length of the dimer. For the description of graphene on metal surfaces, we optimized the basis set for $\mathrm{C}$ using graphene with the experimental lattice constant $(2.46 \AA)$. The mesh size of the grid is controlled by an energy cutoff which sets the wavelength of the shortest plane wave that can be represented on the grid; here we use a cutoff value of 300 Ry. For the Monkhorst-Pack $k$-point meshes we are using a cutoff of $30 \AA$, which is found to be enough for the convergence of all systems under consideration.

For the investigation of the adsorption of noble gases on (111) metal surfaces, we have modeled slabs using a periodically repeated hexagonal supercell with a $(\sqrt{3} \times \sqrt{3}) R 30^{\circ}$ structure made of 18 metal atoms distributed over 6 layers and separated along the direction orthogonal to the surface by a vacuum region of about $20 \AA$, in conjunction with dipole corrections [107]. The topmost 2 layers were relaxed for the clean slab with the relaxed bulk geometry. Then the RG monolayer was relaxed on top of the slab with a fixed geometry for atop sites. The adsorption energy of the RG monolayer on a metal surface is given by

$$
E_{\mathrm{ads}}^{\mathrm{mon}}=E_{\mathrm{tot}}-E_{\mathrm{slab}}-E_{\mathrm{mon}},
$$

where $E_{\text {tot }}, E_{\text {slab }}$, and $E_{\text {mon }}$ represent the energy of the whole system (RG monolayer + metal surface), the bare metal slab energy, and the RG monolayer energy, respectively. One should note that the experimental adsorption energy $E_{\text {ads }}^{\text {mon }}$ includes not only the interaction of RG atoms with the substrate but also lateral vdW RG-RG interactions [108]. In order to exclude the latter interaction, we define the adsorption of separate $\mathrm{RG}$ atoms as

$$
E_{\text {ads }}^{\mathrm{RG}}=E_{\text {tot }}-E_{\text {slab }}-E_{\mathrm{RG}},
$$

where $E_{\mathrm{RG}}$ is the energy of an isolated (free) RG atom as calculated in a box with dimensions $20 \times 21 \times 22 \AA$. Here we note that when using a localized basis set it is necessary to check the influence of the basis-set superposition error (BSSE). In order to avoid the BSSE, the so-called counterpoise (CP) correction is used [109]. For the adsorption of noble gases on (111) metal surfaces, the BSSE shifts the adsorption energy to a higher value: up to $2-3 \mathrm{meV}$ for $\mathrm{XC}$ functionals without dispersion and up to $1 \mathrm{meV}$ for $\mathrm{XC}$ functionals with dispersion. Since we are focusing on the results of dispersion-corrected XC functionals, the effect of 
TABLE I. Relaxed lattice constants (in $\AA$ ) for bulk Cu, Pt, Pd, and Ag calculated with various XC functionals and compared to experiment. The experimental values are obtained by subtracting the zero-point anharmonic expansion (ZPAE) from the experimental zero-temperature values $[111,112]$.

\begin{tabular}{|c|c|c|c|c|c|c|c|c|}
\hline & Expt. & PBE & SG4 & PBEsol & PBE+VV10L & $\mathrm{SG} 4+\mathrm{VV} 10 \mathrm{~m}$ & PBEsol+VV10s & $v d W-D F-c x$ \\
\hline $\mathrm{Cu}$ & 3.595 & 3.698 & 3.637 & 3.632 & 3.676 & 3.620 & 3.620 & 3.648 \\
\hline $\mathrm{Pt}$ & 3.912 & 4.010 & 3.952 & 3.956 & 3.989 & 3.939 & 3.944 & 3.964 \\
\hline $\mathrm{Ag}$ & 4.062 & 4.194 & 4.103 & 4.099 & 4.154 & 4.077 & 4.077 & 4.104 \\
\hline MAE & & 0.11 & 0.04 & 0.04 & 0.08 & 0.02 & 0.02 & 0.05 \\
\hline MARE $(\%)$ & & 2.9 & 1.1 & 1.0 & 2.2 & 0.6 & 0.6 & 1.2 \\
\hline
\end{tabular}

the BSSE can be considered to be negligible. For this reason, the adsorption energies reported in Table II were calculated with no $\mathrm{CP}$ correction.

For the description of the adsorption of graphene on (111) metal surfaces, we use a $(1 \times 1) R 30^{\circ}$ surface unit cell for $\mathrm{Cu}(111)$ and $\mathrm{a}(\sqrt{3} \times \sqrt{3}) R 30^{\circ}$ unit cell for $\mathrm{Pt}(111), \operatorname{Pd}(111)$, and $\operatorname{Ag}(111)$, as they are compatible with the graphene lattice distance. In all calculations, we have used the experimental metal lattice parameter and we have stretched or squeezed the graphene monolayer to match the unit cell, as in the work of Olsen et al. [110]. The metal surfaces were constructed using 6 atomic layers with vacuum space between repeated images of about $20 \AA$. In the case of graphene adsorption on (111) metal surfaces the effect of the BSSE is essential (up to $10 \mathrm{meV}$ ), so we use the $\mathrm{CP}$ correction for the estimation of binding energies, and for the estimation of the equilibrium distance between graphene and metal surfaces the $\mathrm{CP}$ correction is performed for vdW XC functionals.

We note that for $\mathrm{RG}$ atoms basis sets were optimized for dimers while the basis set of carbon was optimized for carbon in graphene. The adsorption of RG atoms on atop sites of metal surfaces corresponds to a dimer interaction resulting in small changes in the electron distribution and giving a negligible BSSE. Instead, the adsorption of graphene on metal surfaces yields a large artificial stabilization describing an electron distribution that results in essential BSSE effects.

For surface-energy calculations the unit cell was composed of periodic six-layer slabs with $(1 \times 1) R 30^{\circ}$ in-plane periodicity and a vacuum space of approximately $10 \AA$. The topmost two layers were relaxed on both sides of the slab, while inner layers kept the relaxed bulk geometry. We obtain the surface energy as follows:

$$
\sigma_{s}=\frac{1}{2}\left(E_{\mathrm{slab}}^{N}-N E_{\mathrm{bulk}}\right),
$$

where $E_{\mathrm{slab}}^{N}$ is the total energy of an $N$-atom slab, $E_{\text {bulk }}$ is the bulk energy per atom, and the factor $1 / 2$ accounts for the two surfaces in the slab unit cell.

We note that the use of 6 atomic layers for the simulations of metal surfaces is well converged for the adsorption (binding) energies within a relative error of less than $1 \%$.

\section{RESULTS}

In Table I, we present the relaxed lattice constants we have obtained for the bulk materials under consideration by using various $\mathrm{XC}$ functionals. The SG4+VV10m and
PBEsol+VV10s functionals yield the relaxed lattice constants that are closest to experiment. Then we have the relaxed lattice constants obtained by using the functionals SG4, PBEsol, and vdW-DF-cx. The worst results are obtained with the use of PBE+VV10L and PBE.

\section{A. RG adsorption on (111) transition-metal surfaces}

The vdW interaction plays an essential role in the adsorption of RG atoms/monolayers on (111) transition-metal surfaces. Such systems have been carefully investigated [113,116-119,121-129], as they represent an important test for XC functionals, even if the experimental data are limited. In Table II, we report the results we have obtained for the RGsurface equilibrium distance $d_{\mathrm{ads}}$, for the adsorption energy of the RG monolayer referring to $E_{\text {ads }}^{\text {mon }}$ defined by Eq. (8), and for the adsorption energy of the RG atom referring to $E_{\mathrm{ads}}^{\mathrm{RG}}$ defined by Eq. (9).

In the case of the equilibrium distance $d_{\mathrm{ads}}$, PBE systematically overestimates this quantity by more than $10 \%$ while PBEsol is accurate especially for the adsorption of Xe on $\mathrm{Cu}(111), \operatorname{Pt}(111), \operatorname{Pd}(111)$, and $\mathrm{Ag}(111)$ surfaces. On the other hand, SG4 largely overestimates this quantity in the case of $\mathrm{Ar}$ on $\mathrm{Cu}(111)$ and $\mathrm{Xe}$ on $\mathrm{Ag}(111)$, but it is accurate for the other systems [Xe on $\mathrm{Cu}(111), \operatorname{Pt}(111)$, and $\mathrm{Pd}(111)]$. All vdW-corrected functionals under study, PBE+VV10L, SG4+VV10m, PBEsol+VV10s, as well as vdW-DF-cx, yield reasonably accurate equilibrium distances.

For the adsorption energies, SG4 and PBE fail badly for all systems, while PBEsol gives better results although still considerably away from the experimental values. All dispersion-corrected functionals under consideration yield, however, realistic adsorption energies. With the use, in particular, of PBEsol+VV10s one obtains adsorption energies that are accurate for $\mathrm{Xe}$ on $\mathrm{Cu}(111), \operatorname{Pt}(111)$, and $\mathrm{Pd}(111)$, although adsorption energies are overestimated with the use of this functional in the case of $\mathrm{Ar}$ on $\mathrm{Cu}(111)$ and $\mathrm{Xe}$ on $\mathrm{Ag}(111)$. SG4+VV10m and vdW-DF-cx have the tendency to slightly underestimate all adsorption energies under study.

\section{B. Graphene adsorption on (111) transition-metal surfaces}

There are numerous theoretical and experimental studies of the adsorption of graphene on transition-metal surfaces [130] such as $\operatorname{Pt}(111)$ [131-141], $\operatorname{Pd}(111)$ [57,142-147], $\operatorname{Ag}(111)$ [148-155], and $\mathrm{Cu}(111)$ [156-164]. The ground-state 
TABLE II. Structural parameters determined for the $(\sqrt{3} \times \sqrt{3}) R 30^{\circ}$ structure of RG atoms on (111) close-packed transition-metal surfaces for atop sites. The RG-surface equilibrium distance $d_{\text {ads }}$ is given in $\AA$; the adsorption energy of the RG monolayer $E_{\text {ads }}^{\text {mon }}$ and the adsorption energy of the RG atom $E_{\text {ads }}^{\mathrm{RG}}$ are given in meV. Reasonable theoretical values (with a maximum deviation of $5 \%$ from experiment) are boldfaced.

\begin{tabular}{|c|c|c|c|c|c|c|c|c|}
\hline & Expt. & PBE & SG4 & PBEsol & $\mathrm{PBE}+\mathrm{VV} 10 \mathrm{~L}$ & SG4+VV10m & PBEsol+VV10s & vdW-DF-c \\
\hline \multicolumn{9}{|l|}{$\mathrm{Ar}$ on $\mathrm{Cu}(111)$} \\
\hline$d_{\mathrm{ads}}$ & $3.53^{\mathrm{a}}$ & 4.252 & 5.286 & 3.783 & 3.679 & 3.641 & 3.492 & 3.749 \\
\hline$E_{\mathrm{ads}}^{\mathrm{mon}}$ & & -13.29 & -5.05 & -16.77 & -76.56 & -97.24 & -51.53 & -97.48 \\
\hline $\begin{array}{l}E_{\mathrm{ads}}^{\mathrm{RG}} \\
\mathrm{Xe} \text { on } \mathrm{Cu}(111)\end{array}$ & $-84.8^{\mathrm{a}},-90.7^{\mathrm{a}}$ & -25.21 & -9.23 & -25.27 & -100.39 & -127.14 & -65.25 & -141.94 \\
\hline$d_{\mathrm{ads}}$ & $3.6^{\mathrm{a}}, 3.52-3.68^{\mathrm{bc}}$ & 4.351 & 3.654 & 3.557 & 3.663 & 3.388 & 3.376 & 3.564 \\
\hline$E_{\mathrm{ads}}^{\mathrm{mon}}$ & $-190^{\mathrm{b}},-200^{\mathrm{cd}}$ & -17.19 & -8.7 & -55.34 & -140.03 & -192.68 & -150.8 & -181.89 \\
\hline$E_{\mathrm{ads}}^{\mathrm{RG}}$ & -174 to $-193^{a}$ & -24.4 & -24.54 & -67.39 & -189.53 & -218.19 & -189.33 & -250.88 \\
\hline \multicolumn{9}{|l|}{$\mathrm{Xe}$ on $\mathrm{Pt}(111)$} \\
\hline$d_{\mathrm{ads}}$ & $3.3-3.5^{\mathrm{d}}$ & 3.878 & 3.347 & 3.281 & 3.431 & 3.174 & 3.16 & 3.346 \\
\hline$E_{\text {ads }}^{\text {mon }}$ & $-270^{\mathrm{bc}},-260$ to $-280^{\mathrm{e}}$ & -31.28 & -37.45 & -115.02 & -212.9 & -272.96 & -250.27 & -253.88 \\
\hline$E_{\mathrm{ads}}^{\mathrm{RG}}$ & $-274^{\mathrm{a}}$ & -47.98 & -28.26 & -130.2 & -260.05 & -311.2 & -282.53 & -330.94 \\
\hline \multicolumn{9}{|l|}{ Xe on $\operatorname{Pd}(111)$} \\
\hline$d_{\mathrm{ads}}$ & $3.01-3.13^{\mathrm{cd}}$ & 3.405 & 3.047 & 3.035 & 3.182 & 2.985 & 2.96 & 3.102 \\
\hline$E_{\mathrm{ads}}^{\mathrm{mon}}$ & $-330^{\mathrm{c}},-320^{\mathrm{d}}$ & -61.9 & -120.92 & -195.33 & -256.54 & -356.19 & -342.21 & -315.85 \\
\hline$E_{\mathrm{ads}}^{\mathrm{RG}}$ & $-360^{\mathrm{c}},-265$ to $-275^{\mathrm{a}}$ & -78.52 & -110.13 & -210.75 & -304.54 & -393.26 & -376.79 & -393.35 \\
\hline $\mathrm{Xe}$ on $\operatorname{Ag}(111)$ & \multicolumn{8}{|c|}{ (Incommensurate structure, mixed site) } \\
\hline$d_{\mathrm{ads}}$ & $3.55-3.65^{\mathrm{a}}$ & 4.527 & 5.741 & 3.652 & 3.761 & 3.533 & 3.446 & 3.693 \\
\hline$E_{\text {ads }}^{\text {mon }}$ & $-225^{\mathrm{bcf}}$ & -15.41 & -3.74 & -43.69 & -130.48 & -166.91 & -135.37 & -163.5 \\
\hline$E_{\mathrm{ads}}^{\mathrm{RG}}$ & $-225^{\mathrm{bcf}},-196$ to $-226^{\mathrm{a}}$ & -31.15 & -0.55 & -57.43 & -171.79 & -205.38 & -163.45 & -236.88 \\
\hline
\end{tabular}

${ }^{\mathrm{a}}$ Reference [113]; incommensurate or unknown structure.

${ }^{\mathrm{b}}$ Reference [114].

${ }^{\mathrm{c}}$ Reference [115].

${ }^{\mathrm{d}}$ Reference [116].

${ }^{\mathrm{e}}$ Reference [117].

${ }^{\mathrm{f}}$ Reference [120].

properties of these systems have been investigated using RPA $[110,148]$, which is a high-level method accurate for the description of vdW interactions in solids and extended systems $[165,166]$. RPA is, however, less accurate in the case of strong noncovalent interactions due to its inaccurate dynamical correlation part $[11,167]$. RPA benchmark calculations should, therefore, be treated with caution, as argued in Ref. [49] for the case of layered materials.

In Table III, we show the equilibrium distance between graphene and the corresponding metal surface, as obtained with the use of various XC functionals. Note that the graphene lattice constant has been fixed to match the experimental transition-metal one (see Table I of Ref. [110]). This fact may influence the binding energy between the graphene and the metal surface, but it should not have an impact on the equilibrium distance $d_{\text {ads }}$.

We note that the BSSE creates an artificial attraction between the graphene layer and the metal surface, which reduces the equilibrium distance $d_{\text {ads }}$. The counterpoise correction eliminates this error, but it tends to overestimate $d_{\text {ads }}$. Hence, the true value for $d_{\mathrm{ads}}$ is somewhere in between. For this reason, for the results we have obtained by using dispersioncorrected XC functionals we put in Table III the averaged equilibrium distance with the corresponding error bar. Whenever XC functionals are used with no dispersion, however, we do not consider the $\mathrm{CP}$ correction so the equilibrium distances can be slightly underestimated. For the evaluation of binding energies (see Table IV), we simply do not take into account

TABLE III. Equilibrium distances $d_{\text {ads }}$ (in $\AA$ ) of graphene on transition-metal (111) surfaces, as obtained with the use of various XC functionals.

\begin{tabular}{|c|c|c|c|c|c|c|c|c|c|}
\hline & $\mathrm{RPA}^{\mathrm{a}}$ & PBE & SG4 & PBEsol & $\mathrm{PBE}+\mathrm{VV10L}$ & $\mathrm{SG} 4+\mathrm{VV} 10 \mathrm{~m}$ & PBEsol+VV10s & vdW-DF-cx & Expt. \\
\hline $\mathrm{Cu}$ & 3.09 & 3.91 & 3.25 & 3.28 & $3.43 \pm 0.04$ & $2.94 \pm 0.04$ & $3.04 \pm 0.02$ & $3.16 \pm 0.02$ & \\
\hline $\mathrm{Pt}$ & 3.42 & 3.86 & 3.50 & 3.47 & $3.54 \pm 0.05$ & $3.32 \pm 0.04$ & $3.31 \pm 0.04$ & $3.34 \pm 0.04$ & $3.3^{\mathrm{b}}$ \\
\hline $\mathrm{Pd}$ & 3.34 & 3.71 & 3.25 & 3.25 & $3.36 \pm 0.05$ & $3.02 \pm 0.04$ & $3.02 \pm 0.04$ & $3.10 \pm 0.03$ & \\
\hline $\mathrm{Ag}$ & 3.31 & 4.11 & 3.45 & 3.42 & $3.51 \pm 0.04$ & $3.21 \pm 0.03$ & $3.22 \pm 0.03$ & $3.26 \pm 0.02$ & $\approx 2.5^{\mathrm{c}}$ \\
\hline
\end{tabular}

\footnotetext{
a Reference [110].

${ }^{\mathrm{b}}$ Reference [145].

${ }^{\mathrm{c}}$ Reference [148].
} 
TABLE IV. Binding energies per $\mathrm{C}$ atom (in $\mathrm{meV}$ ) at the equilibrium distance between graphene and the surface, as obtained with the use of various XC functionals.

\begin{tabular}{|c|c|c|c|c|c|c|c|c|}
\hline & $\mathrm{RPA}^{\mathrm{a}}$ & PBE & SG4 & PBEsol & $\mathrm{PBE}+\mathrm{VV} 10 \mathrm{~L}$ & $\mathrm{SG} 4+\mathrm{VV} 10 \mathrm{~m}$ & PBEsol+VV10s & vdW-DF-cx \\
\hline $\mathrm{Cu}$ & 68 & 2 & 6 & 14 & 45 & 67 & 51 & 64 \\
\hline $\mathrm{Pt}$ & 84 & 8 & 9 & 19 & 57 & 68 & 55 & 69 \\
\hline $\mathrm{Ag}$ & 78 & 2 & 2 & 11 & 42 & 57 & 43 & 57 \\
\hline
\end{tabular}

${ }^{\text {aReference [110]. }}$

$\mathrm{CP}$ corrections because the BSSE is very small in this case (up to $3 \mathrm{meV}$ for $\mathrm{Cu}$ when the SG4+VV10m XC functional is used and up to $0.5 \mathrm{meV}$ for other materials and functionals).

As shown in Table III, the results we obtain with the use of PBE, PBEsol, and SG4 are larger than their RPA counterparts, except in the case of Pd where PBEsol and SG4 calculations are found to be below RPA. Adding the VV10 nonlocal correlation has the effect of shortening the equilibrium distances. We observe that the equilibrium distances we obtain by using PBE+VV10L are still over the RPA equilibrium distances in the case of $\mathrm{Cu}, \mathrm{Pt}$, and $\mathrm{Ag}$, while PBE+VV10L and RPA calculations are very close to each other in the case of $\mathrm{Pd}$. The equilibrium distances we obtain by using other functionals (SG4+VV10m, PBEsol+VV10s, and vdW-DF-cx) are, however, below those obtained in the RPA, except in the case of $\mathrm{Cu}$; in this case, the use of vdW-DF-cx yields an equilibrium distance that is above that obtained in the RPA and the use of PBEsol+VV10s yields an equilibrium distance that is very close to that obtained in the RPA. We note that with the use of SG4+VV10m, PBEsol+VV10s, and vdW-DF-cx equilibrium distances are found to be very close to experiment for $\mathrm{Pt}$ and closer to experiment than RPA equilibrium distances and also closer to experiment than equilibrium distances obtained with the use of other functionals. We recall that the RPA calculations reported in Table III use PBE orbitals [110], which may induce some errors.

The interaction between the graphene monolayer and the metal surface is known to be weak in the case of $\operatorname{Pt}(111)$, $\mathrm{Cu}(111)$, and $\operatorname{Ag}(111)$ [130]. For graphene on $\operatorname{Pd}(111)$, a $0.3 \mathrm{eV}$ band gap has been found from scanning-tunnelingspectroscopy measurements $[130,168]$, which may indicate a strong hybridization. In Table IV, we report the binding energies per $\mathrm{C}$ atom at the equilibrium distance to the surface. All the results we have obtained by using the vdW XC functionals under consideration exhibit a maximum binding energy for $\mathrm{Pd}(111)$, except in the case of PBE+VV10L which
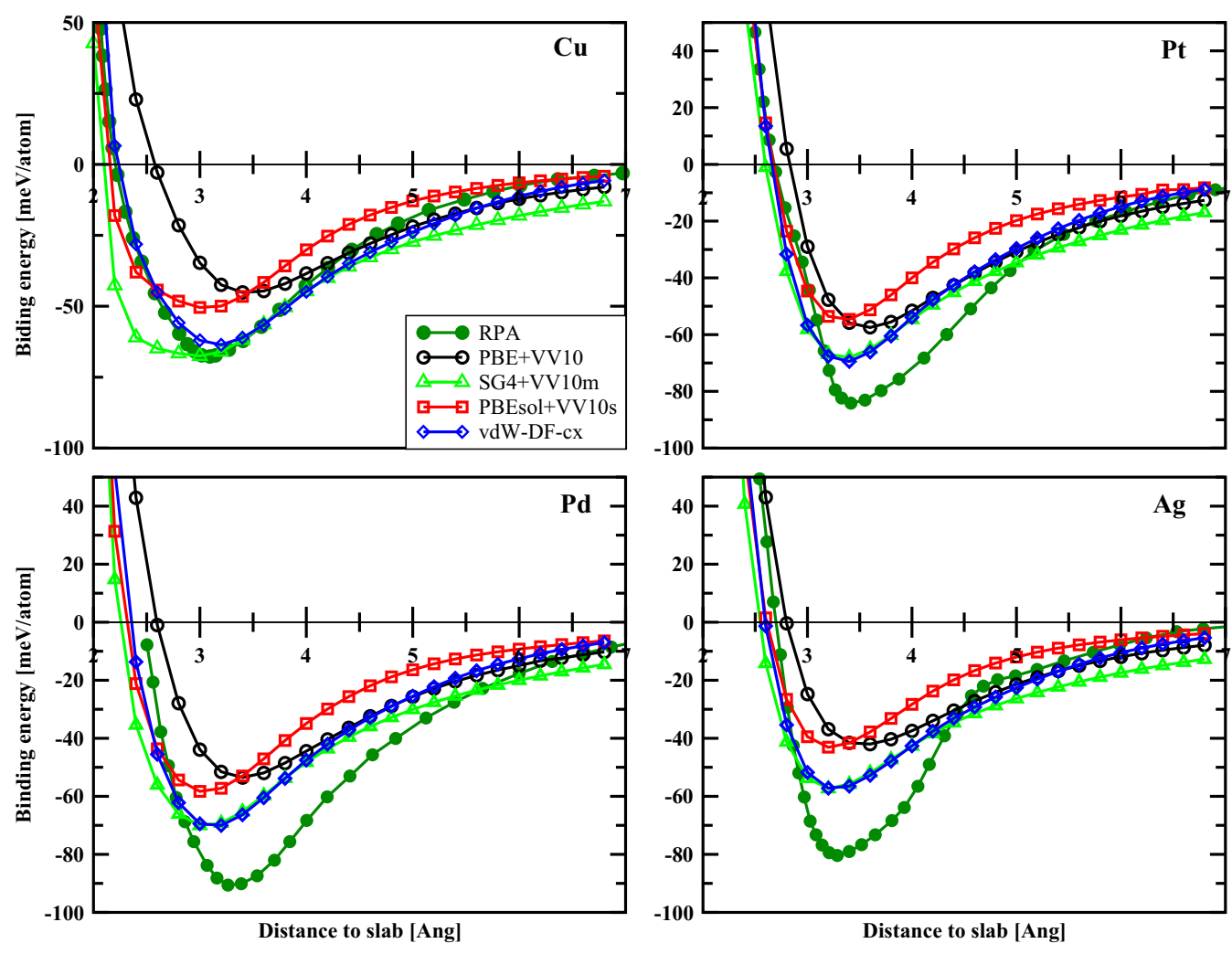

FIG. 3. Potential-energy curves of graphene on various transition-metal (111) surfaces. 
TABLE V. Surface energies (in eV) for $\mathrm{Cu}(111), \mathrm{Pt}(111), \mathrm{Pd}(111)$, and $\mathrm{Ag}(111)$, as obtained with the use of various $\mathrm{XC}$ functionals and compared to experiment. The calculated surface energies closest to experiment are boldfaced.

\begin{tabular}{lcccccccc}
\hline \hline & Expt. $^{a}$ & PBE & SG4 & PBEsol & PBE+VV10L & SG4+VV10m & PBEsol+VV10s & vdW-DF-cx \\
\hline $\mathrm{Cu}$ & 0.65 & 0.47 & 0.58 & 0.61 & 0.57 & 0.7 & $\mathbf{0 . 6 2}$ & 0.58 \\
$\mathrm{Pt}$ & 1.08 & 0.68 & 0.85 & 0.82 & 0.86 & $\mathbf{1 . 0 1}$ & 0.92 & 0.87 \\
$\mathrm{Pd}$ & 0.85 & 0.61 & 0.75 & 0.74 & 0.76 & 0.9 & $\mathbf{0 . 8 3}$ & 0.78 \\
$\mathrm{Ag}$ & 0.58 & 0.34 & 0.45 & 0.45 & 0.47 & $\mathbf{0 . 5 9}$ & 0.52 & 0.49 \\
$\mathrm{MAE}$ & & 0.27 & 0.13 & 0.14 & 0.13 & $\mathbf{0 . 0 4}$ & 0.07 & 0.11 \\
MARE $(\%)$ & & 33.6 & 16.6 & 16.4 & 15.6 & $\mathbf{5 . 4}$ & 8.0 & 13.5 \\
\hline \hline
\end{tabular}

${ }^{\text {a Reference [169]. }}$

gives a $\mathrm{Pd}(111)$ binding energy that is lower than for $\mathrm{Pt}(111)$ by about $4 \mathrm{meV}$.

Comparing the electronic spectra of the (111) surfaces of $\mathrm{Ag}$ and $\mathrm{Cu}$ with and without the graphene monolayer, it has been suggested that the binding energy of graphene on $\operatorname{Ag}(111)$ should be smaller than in the case of $\mathrm{Cu}(111)$ $[148,149]$. Our calculations confirm this trend: with the use of SG4+VV10m, PBEsol+VV10s, and vdW-DF-cx, one obtains $\operatorname{Ag}(111)$ binding energies that are lower than those of $\mathrm{Au}(111)$ by 10,8 , and $7 \mathrm{meV}$, respectively, in reasonable agreement with the vdW-DF-C09 and vdW-DF2-C09 calculations reported in Ref. [57]. Smaller differences are found with the use of PBE+VV10L: the $\mathrm{Ag}(111)$ binding energy is found to be about $3 \mathrm{meV}$ below its $\mathrm{Au}(111)$ counterpart. RPA calculations, however, predict $\operatorname{Ag}(111)$ binding energies that are larger than those of $\mathrm{Au}(111)$ by about $10 \mathrm{meV}$.

An inspection of Tables II and IV shows that SG4+VV10m and vdW-DF-cx yield similar binding energies, both larger than those obtained with the use of the other functionals under study. PBEsol+VV10s and PBE+VV10L also yield similar binding energies, both below the SG4+VV10m and vdWDF-cx binding energies. PBEsol+VV10s, however, gives a better description of the Pd binding energy with respect to its $\mathrm{Pt}$ counterpart and also gives a better description of the $\mathrm{Ag}$ binding energy with respect to its $\mathrm{Cu}$ counterpart.

Finally, we show in Fig. 3 the potential-energy curves of graphene on $\mathrm{Cu}(111), \operatorname{Pt}(111), \operatorname{Pd}(111)$, and $\mathrm{Ag}(111)$. All curves are smooth and all equilibrium minima are well represented, except for $\mathrm{Cu}(111)$ when SG4+VV10m is used; in this case, the potential-energy curve near the minimum appears to be anomalously flat. Overall, qualitative agreement is found with RPA calculations. At large distances, where the vdW interaction dominates, many-body RPA calculations should be expected to be more accurate than the two-body calculations reported here [166].

\section{Surface formation energies of $\mathrm{Cu}(111)$, $\operatorname{Pt}(111), \operatorname{Pd}(111)$, and $\operatorname{Ag}(111)$}

Here we look at surface energies of the (111) surfaces of $\mathrm{Cu}, \mathrm{Pt}, \mathrm{Pd}$, and $\mathrm{Ag}$. Note that the $\mathrm{Cu}, \mathrm{Pt}, \mathrm{Pd}$, and $\mathrm{Ag}$ transition metals all have a face-centered cubic (fcc) crystal structure, so the (111) surface configuration happens to be the most stable. Thus, it seems reasonable to compare our theoretical calculations to experimental values, as in Refs. [169-171]. Our calculations are presented in Table V. The results we have obtained with the use of SG4 and PBEsol exhibit similar accuracy, both about twice better than with the use of PBE but still underestimating the experiment. We recall that the use of SG4 and PBEsol also yields good surface energies in a jellium model $[172,173]$, which is expected to be realistic for the description of simple metal surfaces.

Introducing a VV10 kernel has the effect of increasing the surface energies. The results we have obtained by using PBE +VV10L are still similar to those obtained with the use of SG4 and PBEsol, vdW-DF-cx calculations are slightly better, and both SG4+VV10m and PBEsol+VV10s are found to perform extremely well, at the level of SCAN+rVV10 [174], exhibiting a good agreement with experiment within an error bar of no more than $10 \%$.

\section{SUMMARY AND CONCLUSIONS}

We have tested several recently developed vdW XC functionals (PBE+VV10L [42], SG4+VV10m [43], and PBEsol+VV10s [49]), together with the popular vdW-DF-cx [45], for clean (111) surfaces of transition metals $\mathrm{Cu}, \mathrm{Pt}, \mathrm{Pd}$, and $\mathrm{Ag}$ and also for the interaction of these surfaces with RG atoms/monolayers and a graphene monolayer. These are emblematic systems in the field of hybrid interfaces connected by weak interactions.

In the case of RG atom/monolayer adsorption on $\mathrm{Cu}(111)$, $\operatorname{Pt}(111), \operatorname{Pd}(111)$, and $\operatorname{Ag}(111)$ surfaces, we have found that all dispersion-corrected $\mathrm{XC}$ functionals perform reasonably well, greatly improving over the corresponding dispersionuncorrected GGA XC functionals. The best performance has been found with the use of PBEsol+VV10s, which yields an accurate prediction of both the RG-surface equilibrium distance and the adsorption energy in most systems under consideration. With the use of PBE+VV10L, however, adsorption energies are generally slightly overestimated, and with the use of SG4+VV10m and vdW-DF-cx one finds adsorption energies that are too low.

In the case of graphene on $\mathrm{Cu}(111), \operatorname{Pt}(111), \operatorname{Pd}(111)$, and $\operatorname{Ag}(111)$ surfaces, all vdW kernels under study yield smooth potential-energy curves, with well-defined equilibrium minima, that are found to be in qualitative agreement with RPA calculations, with the exception of $\mathrm{Cu}(111)$ when SG4+VV10m is used. For equilibrium distances, PBE + VV10L is found to overestimate RPA calculations, while the other dispersion-corrected functionals (SG4+VV10m, PBEsol+VV10s, and vdW-DF-cx) are found to predict equilibrium distances that are lower than their RPA counterparts [except in the case of $\mathrm{Cu}(111)$ where the use 
of PBEsol+VV10s yields and equilibrium distance that is similar to RPA and the use of vdW-DF-cx yields an equilibrium distance that is too high]. In particular, in the case of Pt(111) and $\mathrm{Ag}(111)$, SG4+VV10m, PBEsol+VV10s, and vdW-DF-cx yield equilibrium distances that are lower than their RPA counterparts but closer to experiment. For the binding energies, all functionals under study yield values that are below their RPA counterparts. It is important to note, however, that the use of SG4+VV10m, PBEsol+VV10s, and vdW-DF-cx yields a correct trend: the maximum binding energy is found to occur in the case of $\operatorname{Pd}(111)$, as occurs with the experimental measurements, and the binding energy of graphene on $\operatorname{Ag}(111)$ is found to be lower than on $\mathrm{Cu}(111)$, also as in experiment.

Finally, we have found that the clean (111) surfaces of $\mathrm{Cu}, \mathrm{Pt}, \mathrm{Pd}$, and $\mathrm{Ag}$ are best described with the use of
SG4+VV10m and PBEsol+VV10s, as they both yield extremely accurate surface energies with a mean relative error of less than $10 \%$.

We have used the recently developed PBE+VV10L, SG4+VV10m, and PBEsol+VV10s vdW XC functionals in the framework of the $a b$ initio SIESTA method, thus using a localized basis set, and we conclude that these functionals are indeed very valuable for the investigation of hybrid interfaces connected by weak interactions.

\section{ACKNOWLEDGMENTS}

A.V.T. acknowledges Dr. Federico Marchesin for fruitful discussion. E.A. would like to acknowledge financial support from MINECO-Spain through Plan Nacional Grant No. FIS2015-64886.
[1] W. Kohn and L. J. Sham, Phys. Rev. 140, A1133 (1965).

[2] P. Hohenberg and W. Kohn, Phys. Rev. 136, B864 (1964).

[3] G. E. Scuseria and V. N. Staroverov, in Theory and Application of Computational Chemistry: The First 40 Years, edited by C. E. Dykstra, G. Frenking, K. S. Kim, and G. E. Scuseria (Elsevier, Amsterdam, 2005), pp. 669-724.

[4] F. Della Sala, E. Fabiano, and L. A. Constantin, Int. J. Quantum Chem. 116, 1641 (2016).

[5] E. Fabiano, P. E. Trevisanutto, A. Terentjevs, and L. A. Constantin, J. Chem. Theory Comput. 10, 2016 (2014).

[6] J. Tao, I. W. Bulik, and G. E. Scuseria, Phys. Rev. B 95, 125115 (2017).

[7] J. Sun, B. Xiao, Y. Fang, R. Haunschild, P. Hao, A. Ruzsinszky, G. I. Csonka, G. E. Scuseria, and J. P. Perdew, Phys. Rev. Lett. 111, 106401 (2013).

[8] K. Burke, J. Chem. Phys. 136, 150901 (2012).

[9] A. Landa, P. Soederlind, I. Naumov, J. Klepeis, and L. Vitos, Computation 6, 29 (2018).

[10] L. A. Constantin, E. Fabiano, J. M. Pitarke, and F. Della Sala, Phys. Rev. B 93, 115127 (2016).

[11] L. A. Constantin, Phys. Rev. B 93, 121104(R) (2016).

[12] L. A. Constantin, Phys. Rev. B 78, 155106 (2008).

[13] A. D. Kaplan, K. Wagle, and J. P. Perdew, Phys. Rev. B 98, 085147 (2018).

[14] K. D. Sen, Electronic Structure of Quantum Confined Atoms and Molecules (Springer, Cham, Switzerland, 2014).

[15] M. Shahbaz and K. Szalewicz, Phys. Rev. Lett. 121, 113402 (2018).

[16] S. Grimme, J. Comput. Chem. 27, 1787 (2006).

[17] S. Grimme, J. Antony, S. Ehrlich, and H. Krieg, J. Chem. Phys. 132, 154104 (2010).

[18] R. Sure, J. Antony, and S. Grimme, J. Phys. Chem. B 118, 3431 (2014)

[19] A. Tkatchenko, R. A. DiStasio, Jr., R. Car, and M. Scheffler, Phys. Rev. Lett. 108, 236402 (2012).

[20] M. A. Caro, arXiv:1704.00761.

[21] R. A. DiStasio Jr., V. V. Gobre, and A. Tkatchenko, J. Phys.: Condens. Matter 26, 213202 (2014).

[22] J. Moellmann and S. Grimme, J. Phys. Chem. C 118, 7615 (2014)
[23] T. Bučko, S. Lebègue, J. Hafner, and J. G. Angyan, Phys. Rev. B 87, 064110 (2013).

[24] E. R. Johnson and A. D. Becke, J. Chem. Phys. 124, 174104 (2006).

[25] Q. Gu, P. Lu, K. Xia, J. Sun, and D. Xing, Phys. Rev. B 96, 064517 (2017).

[26] A. Rybakov, A. Larin, and D. Vercauteren, Phys. Chem. Chem. Phys. 19, 20930 (2017).

[27] R. Agbaoye, P. Adebambo, J. Akinlami, T. Afolabi, S. Z. Karazhanov, D. Ceresoli, and G. Adebayo, Comput. Mater. Sci. 139, 234 (2017).

[28] A. M. Murcia Rios, D. N. Komsa, and V. N. Staroverov, J. Phys. Chem. C 122, 14781 (2018).

[29] B. N. Feigelson, V. M. Bermudez, J. K. Hite, Z. R. Robinson, V. D. Wheeler, K. Sridhara, and S. C. Hernández, Nanoscale 7, 3694 (2015).

[30] M. Dion, H. Rydberg, E. Schröder, D. C. Langreth, and B. I. Lundqvist, Phys. Rev. Lett. 92, 246401 (2004).

[31] H. Rydberg, B. I. Lundqvist, D. C. Langreth, and M. Dion, Phys. Rev. B 62, 6997 (2000).

[32] R. H. French, V. A. Parsegian, R. Podgornik, R. F. Rajter, A. Jagota, J. Luo, D. Asthagiri, M. K. Chaudhury, Y. M. Chiang, S. Granick et al., Rev. Mod. Phys. 82, 1887 (2010).

[33] D. C. Langreth, M. Dion, H. Rydberg, E. Schröder, P. Hyldgaard, and B. I. Lundqvist, Int. J. Quantum Chem. 101, 599 (2005).

[34] H. Rydberg, M. Dion, N. Jacobson, E. Schröder, P. Hyldgaard, S. I. Simak, D. C. Langreth, and B. I. Lundqvist, Phys. Rev. Lett. 91, 126402 (2003).

[35] P. Hyldgaard, K. Berland, and E. Schröder, Phys. Rev. B 90 , 075148 (2014).

[36] O. A. Vydrov and T. Van Voorhis, J. Chem. Phys. 133, 244103 (2010).

[37] O. A. Vydrov and T. Van Voorhis, Fundamentals of TimeDependent Density Functional Theory (Springer, Berlin, Heidelberg, 2012), pp. 443-456.

[38] G. Román-Pérez and J. M. Soler, Phys. Rev. Lett. 103, 096102 (2009).

[39] R. Sabatini, T. Gorni, and S. de Gironcoli, Phys. Rev. B 87, 041108(R) (2013). 
[40] T. Björkman, Phys. Rev. B 86, 165109 (2012).

[41] H. Peng, Z.-H. Yang, J. P. Perdew, and J. Sun, Phys. Rev. X 6, 041005 (2016).

[42] H. Peng and J. P. Perdew, Phys. Rev. B 95, 081105(R) (2017).

[43] A. V. Terentjev, P. Cortona, L. A. Constantin, J. M. Pitarke, F. Della Sala, and E. Fabiano, Computation 6, 7 (2018).

[44] V. R. Cooper, Phys. Rev. B 81, 161104(R) (2010).

[45] K. Berland and P. Hyldgaard, Phys. Rev. B 89, 035412 (2014).

[46] K. Berland, C. A. Arter, V. R. Cooper, K. Lee, B. I. Lundqvist, E. Schröder, T. Thonhauser, and P. Hyldgaard, J. Chem. Phys. 140, 18A539 (2014).

[47] T. Thonhauser, S. Zuluaga, C. A. Arter, K. Berland, E. Schröder, and P. Hyldgaard, Phys. Rev. Lett. 115, 136402 (2015).

[48] Y. Jiao, E. Schröder, and P. Hyldgaard, Phys. Rev. B 97, 085115 (2018).

[49] A. V. Terentjev, L. A. Constantin, and J. M. Pitarke, Phys. Rev. B 98, 214108 (2018).

[50] I. Hamada, Phys. Rev. B 89, 121103(R) (2014).

[51] T. Björkman, J. Chem. Phys. 141, 074708 (2014).

[52] N. Mounet, M. Gibertini, P. Schwaller, D. Campi, A. Merkys, A. Marrazzo, T. Sohier, I. E. Castelli, A. Cepellotti, G. Pizzi et al., Nat. Nanotechnol. 13, 246 (2018).

[53] G. Graziano, J. Klimeš, F. Fernandez-Alonso, and A. Michaelides, J. Phys.: Condens. Matter 24, 424216 (2012).

[54] T. Bucko, J. Hafner, S. Lebegue, and J. G. Angyán, J. Phys. Chem. A 114, 11814 (2010).

[55] A. Marini, P. García-González, and A. Rubio, Phys. Rev. Lett. 96, 136404 (2006).

[56] S. A. Tawfik, T. Gould, C. Stampfl, and M. J. Ford, Phys. Rev. Mater. 2, 034005 (2018).

[57] I. Hamada and M. Otani, Phys. Rev. B 82, 153412 (2010).

[58] I. Hamada, Phys. Rev. B 86, 195436 (2012).

[59] K. Berland and P. Hyldgaard, Phys. Rev. B 87, 205421 (2013).

[60] R. E. Mapasha, A. M. Ukpong, and N. Chetty, Phys. Rev. B 85, 205402 (2012).

[61] T. Gould, S. Lebègue, J. G. Ángyán, and T. Bučko, J. Chem. Theory Comput. 12, 5920 (2016).

[62] F. Tran, L. Kalantari, B. Traoré, X. Rocquefelte, and P. Blaha, Phys. Rev. Mater. 3, 063602 (2019).

[63] F. Tran and J. Hutter, J. Chem. Phys. 138, 204103 (2013).

[64] P. L. Silvestrelli and A. Ambrosetti, J. Chem. Phys. 140, 124107 (2014).

[65] A. Ambrosetti and P. L. Silvestrelli, Carbon 139, 486 (2018).

[66] A. Ambrosetti and P. L. Silvestrelli, J. Phys. Chem. Lett. 10, 2044 (2019)

[67] J. F. Dobson, A. White, and A. Rubio, Phys. Rev. Lett. 96, 073201 (2006).

[68] A. Ruzsinszky, J. P. Perdew, J. Tao, G. I. Csonka, and J. M. Pitarke, Phys. Rev. Lett. 109, 233203 (2012).

[69] A. Ambrosetti, N. Ferri, R. A. DiStasio, and A. Tkatchenko, Science 351, 1171 (2016).

[70] A. Ambrosetti, P. L. Silvestrelli, and A. Tkatchenko, Phys. Rev. B 95, 235417 (2017).

[71] J. Harris and A. Griffin, Phys. Rev. B 11, 3669 (1975).

[72] D. C. Langreth and J. P. Perdew, Phys. Rev. B 15, 2884 (1977).

[73] J. Toulouse, I. C. Gerber, G. Jansen, A. Savin, and J. G. Angyán, Phys. Rev. Lett. 102, 096404 (2009).

[74] J. F. Dobson, J. Wang, and T. Gould, Phys. Rev. B 66, 081108(R) (2002).
[75] L. A. Constantin and J. M. Pitarke, Phys. Rev. B 75, 245127 (2007).

[76] A. V. Terentjev, L. A. Constantin, and J. M. Pitarke, Phys. Rev. B 98, 085123 (2018).

[77] M. Corradini, R. Del Sole, G. Onida, and M. Palummo, Phys. Rev. B 57, 14569 (1998).

[78] J. Toulouse, Phys. Rev. B 72, 035117 (2005).

[79] C. F. Richardson and N. W. Ashcroft, Phys. Rev. B 50, 8170 (1994).

[80] J. E. Bates, S. Laricchia, and A. Ruzsinszky, Phys. Rev. B 93, 045119 (2016).

[81] J. E. Bates, J. Sensenig, and A. Ruzsinszky, Phys. Rev. B 95, 195158 (2017).

[82] A. Ruzsinszky, L. A. Constantin, and J. M. Pitarke, Phys. Rev. B 94, 165155 (2016).

[83] J. F. Dobson and J. Wang, Phys. Rev. B 62, 10038 (2000).

[84] A. Görling, Int. J. Quantum Chem. 69, 265 (1998).

[85] Y.-H. Kim and A. Görling, Phys. Rev. Lett. 89, 096402 (2002).

[86] J. Erhard, P. Bleiziffer, and A. Görling, Phys. Rev. Lett. 117, 143002 (2016).

[87] C. E. Patrick and K. S. Thygesen, J. Chem. Phys. 143, 102802 (2015)

[88] K. Pernal, Phys. Rev. Lett. 120, 013001 (2018).

[89] L. A. Constantin and J. M. Pitarke, Phys. Rev. B 83, 075116 (2011).

[90] K. Rapcewicz and N. W. Ashcroft, Phys. Rev. B 44, 4032 (1991).

[91] J. M. Soler, E. Artacho, J. D. Gale, A. García, J. Junquera, P. Ordejón, and D. Sánchez-Portal, J. Phys.: Condens. Matter 14, 2745 (2002).

[92] P. Giannozzi, S. Baroni, N. Bonini, M. Calandra, R. Car, C. Cavazzoni, D. Ceresoli, G. L. Chiarotti, M. Cococcioni, I. Dabo et al., J. Phys.: Condens. Matter 21, 395502 (2009).

[93] D. C. Langreth and S. H. Vosko, Adv. Quantum Chem. 21, 175 (1990).

[94] P. R. Antoniewicz and L. Kleinman, Phys. Rev. B 31, 6779 (1985).

[95] J. P. Perdew, K. Burke, and M. Ernzerhof, Phys. Rev. Lett. 77, 3865 (1996).

[96] P. Elliott, D. Lee, A. Cangi, and K. Burke, Phys. Rev. Lett. 100, 256406 (2008).

[97] P. Elliott and K. Burke, Can. J. Chem. 87, 1485 (2009).

[98] L. A. Constantin, J. C. Snyder, J. P. Perdew, and K. Burke, J. Chem. Phys. 133, 241103 (2010).

[99] E. Fabiano and L. A. Constantin, Phys. Rev. A 87, 012511 (2013).

[100] E. H. Lieb and S. Oxford, Int. J. Quantum Chem. 19, 427 (1981).

[101] L. A. Constantin, A. Terentjevs, F. Della Sala, and E. Fabiano, Phys. Rev. B 91, 041120(R) (2015).

[102] O. A. Vydrov and T. Van Voorhis, Phys. Rev. A 81, 062708 (2010).

[103] P. Jurečka, J. Šponer, J. Černý, and P. Hobza, Phys. Chem. Chem. Phys. 8, 1985 (2006).

[104] T. Takatani, E. G. Hohenstein, M. Malagoli, M. S. Marshall, and C. D. Sherrill, J. Chem. Phys. 132, 144104 (2010).

[105] N. Troullier and J. L. Martins, Phys. Rev. B 43, 1993 (1991).

[106] S. G. Louie, S. Froyen, and M. L. Cohen, Phys. Rev. B 26, 1738 (1982).

[107] L. Bengtsson, Phys. Rev. B 59, 12301 (1999). 
[108] X. Sun and Y. Yamauchi, J. Appl. Phys. 110, 103701 (2011).

[109] S. Boys and F. Bernardi, Mol. Phys. 100, 65 (2002).

[110] T. Olsen and K. S. Thygesen, Phys. Rev. B 87, 075111 (2013).

[111] J. Sun, M. Marsman, G. I. Csonka, A. Ruzsinszky, P. Hao, Y. S. Kim, G. Kresse, and J. P. Perdew, Phys. Rev. B 84, 035117 (2011).

[112] P. Janthon, S. Luo, S. M. Kozlov, F. Vines, J. Limtrakul, D. G. Truhlar, and F. Illas, J. Chem. Theory Comput. 10, 3832 (2014).

[113] G. Vidali, G. Ihm, H.-Y. Kim, and M. W. Cole, Surf. Sci. Rep. 12, 135 (1991).

[114] T. Seyller, M. Caragiu, R. Diehl, P. Kaukasoina, and M. Lindroos, Chem. Phys. Lett. 291, 567 (1998).

[115] M. Caragiu, T. Seyller, and R. D. Diehl, Phys. Rev. B 66, 195411 (2002).

[116] R. Diehl, T. Seyller, M. Caragiu, G. Leatherman, N. Ferralis, K. Pussi, P. Kaukasoina, and M. Lindroos, J. Phys.: Condens. Matter 16, S2839 (2004).

[117] V. G. Ruiz, W. Liu, E. Zojer, M. Scheffler, and A. Tkatchenko, Phys. Rev. Lett. 108, 146103 (2012).

[118] D.-L. Chen, W. Al-Saidi, and J. K. Johnson, J. Phys.: Condens. Matter 24, 424211 (2012).

[119] P. L. Silvestrelli, A. Ambrosetti, S. Grubisiĉ, and F. Ancilotto, Phys. Rev. B 85, 165405 (2012).

[120] N. Ferralis, H. Li, K. Hanna, J. Stevens, H. Shin, F. Pan, and R. Diehl, J. Phys.: Condens. Matter 19, 056011 (2007).

[121] J. P. P. Ramalho, J. R. Gomes, and F. Illas, RSC Adv. 3, 13085 (2013).

[122] J. L. F. Da Silva, C. Stampfl, and M. Scheffler, Phys. Rev. Lett. 90, 066104 (2003).

[123] J. L. F. Da Silva, C. Stampfl, and M. Scheffler, Phys. Rev. B 72, 075424 (2005).

[124] J. Tao and A. M. Rappe, Phys. Rev. Lett. 112, 106101 (2014).

[125] P. L. Silvestrelli and A. Ambrosetti, Phys. Rev. B 91, 195405 (2015).

[126] A. Betancourt and D. Bird, J. Phys.: Condens. Matter 12, 7077 (2000).

[127] V. G. Ruiz, W. Liu, and A. Tkatchenko, Phys. Rev. B 93, 035118 (2016).

[128] P. Lazić, Ž. Crljen, R. Brako, and B. Gumhalter, Phys. Rev. B 72, 245407 (2005).

[129] D.-L. Chen, W. A. Al-Saidi, and J. K. Johnson, Phys. Rev. B 84, 241405(R) (2011).

[130] M. Batzill, Surf. Sci. Rep. 67, 83 (2012).

[131] A. J. Martínez-Galera, N. Nicoara, J. I. Martínez, Y. J. Dappe, J. Ortega, and J. M. Gómez-Rodríguez, J. Phys. Chem. C 118, 12782 (2014)

[132] A. Politano, A. Marino, V. Formoso, and G. Chiarello, AIP Adv. 1, 042130 (2011)

[133] P. Sutter, J. T. Sadowski, and E. Sutter, Phys. Rev. B 80, 245411 (2009).

[134] T. Fujita, W. Kobayashi, and C. Oshima, Surf. Interface Anal. 37, 120 (2005).

[135] M. Gao, Y. Pan, L. Huang, H. Hu, L. Zhang, H. Guo, S. Du, and H.-J. Gao, Appl. Phys. Lett. 98, 033101 (2011).

[136] B. J. Kang, J. H. Mun, C. Y. Hwang, and B. J. Cho, J. Appl. Phys. 106, 104309 (2009).

[137] G. Otero, C. González, A. L. Pinardi, P. Merino, S. Gardonio, S. Lizzit, M. Blanco-Rey, K. Van de Ruit, C. F. J. Flipse, J. Méndez et al., Phys. Rev. Lett. 105, 216102 (2010).
[138] A. Politano, A. R. Marino, V. Formoso, D. Farías, R. Miranda, and G. Chiarello, Phys. Rev. B 84, 033401 (2011).

[139] N. A. Vinogradov, K. Schulte, M. L. Ng, A. Mikkelsen, E. Lundgren, N. Martensson, and A. Preobrajenski, J. Phys. Chem. C 115, 9568 (2011).

[140] Y. Yamada, C. Sugawara, Y. Satake, Y. Yokoyama, R. Okada, T. Nakayama, M. Sasaki, T. Kondo, J. Oh, J. Nakamura et al., J. Phys.: Condens. Matter 22, 304010 (2010).

[141] A. Politano, A. R. Marino, V. Formoso, and G. Chiarello, Carbon 50, 734 (2012).

[142] P. A. Khomyakov, G. Giovannetti, P. C. Rusu, G. Brocks, J. van den Brink, and P. J. Kelly, Phys. Rev. B 79, 195425 (2009).

[143] M. Andersen, L. Hornekær, and B. Hammer, Phys. Rev. B 86, 085405 (2012)

[144] Q.-S. Chen, Z.-N. Xu, S.-Y. Peng, Y.-M. Chen, D.-M. Lv, Z.Q. Wang, J. Sun, and G.-C. Guo, J. Power Sources 282, 471 (2015).

[145] M. Vanin, J. J. Mortensen, A. K. Kelkkanen, J. M. GarciaLastra, K. S. Thygesen, and K. W. Jacobsen, Phys. Rev. B 81, 081408(R) (2010).

[146] H. Mok, A. Ebnonnasir, Y. Murata, S. Nie, K. McCarty, C. Ciobanu, and S. Kodambaka, Appl. Phys. Lett. 104, 101606 (2014).

[147] B. Wang, M. Caffio, C. Bromley, H. Früchtl, and R. Schaub, ACS Nano 4, 5773 (2010).

[148] I. Lončarić and V. Despoja, Phys. Rev. B 90, 075414 (2014).

[149] B. Kiraly, E. V. Iski, A. J. Mannix, B. L. Fisher, M. C. Hersam, and N. P. Guisinger, Nat. Commun. 4, 2804 (2013).

[150] J. Tesch, P. Leicht, F. Blumenschein, L. Gragnaniello, M. Fonin, L. E. M. Steinkasserer, B. Paulus, E. Voloshina, and Y. Dedkov, Sci. Rep. 6, 23439 (2016).

[151] Y. Li, W. Zhang, M. Morgenstern, and R. Mazzarello, Phys. Rev. Lett. 110, 216804 (2013).

[152] X. Lin and J. Ni, Phys. Rev. B 86, 075440 (2012).

[153] Z. Li, Z. Yang, S. Qiao, J. Hu, and R. Wu, J. Phys.: Condens. Matter 23, 225502 (2011).

[154] K. Simonov, A. Generalov, A. Vinogradov, G. Svirskiy, A. Cafolla, C. McGuinness, T. Taketsugu, A. Lyalin, N. Mårtensson, and A. Preobrajenski, Sci. Rep. 8, 3506 (2018).

[155] Y. Pan, L. Zhang, L. Huang, L. Li, L. Meng, M. Gao, Q. Huan, X. Lin, Y. Wang, S. Du et al., Small 10, 2215 (2014).

[156] L. Gao, J. R. Guest, and N. P. Guisinger, Nano Lett. 10, 3512 (2010).

[157] H. Vita, S. Böttcher, K. Horn, E. Voloshina, R. Ovcharenko, T. Kampen, A. Thissen, and Y. S. Dedkov, Sci. Rep. 4, 5704 (2014).

[158] Y. Ogawa, B. Hu, C. M. Orofeo, M. Tsuji, K.-i. Ikeda, S. Mizuno, H. Hibino, and H. Ago, J. Phys. Chem. Lett. 3, 219 (2012).

[159] T. Niu, M. Zhou, J. Zhang, Y. Feng, and W. Chen, J. Am. Chem. Soc. 135, 8409 (2013).

[160] I. Wlasny, P. Dabrowski, M. Rogala, P. Kowalczyk, I. Pasternak, W. Strupinski, J. Baranowski, and Z. Klusek, Appl. Phys. Lett. 102, 111601 (2013).

[161] K. M. Reddy, A. D. Gledhill, C.-H. Chen, J. M. Drexler, and N. P. Padture, Appl. Phys. Lett. 98, 113117 (2011).

[162] S. Gottardi, K. Müller, L. Bignardi, J. C. Moreno-López, T. A. Pham, O. Ivashenko, M. Yablonskikh, A. Barinov, J. Bjórk, P. Rudolf et al., Nano Lett. 15, 917 (2015). 
[163] Z. R. Robinson, P. Tyagi, T. R. Mowll, C. A. Ventrice Jr., and J. B. Hannon, Phys. Rev. B 86, 235413 (2012).

[164] M. Ishihara, Y. Koga, J. Kim, K. Tsugawa, and M. Hasegawa, Mater. Lett. 65, 2864 (2011).

[165] S. Lebègue, J. Harl, T. Gould, J. G. Ángyán, G. Kresse, and J. F. Dobson, Phys. Rev. Lett. 105, 196401 (2010).

[166] J. F. Dobson, T. Gould, and G. Vignale, Phys. Rev. X 4, 021040 (2014).

[167] A. Ruzsinszky, J. P. Perdew, and G. I. Csonka, J. Chem. Theory Comput. 6, 127 (2009).

[168] S.-Y. Kwon, C. V. Ciobanu, V. Petrova, V. B. Shenoy, J. Bareño, V. Gambin, I. Petrov, and S. Kodambaka, Nano Lett. 9, 3985 (2009).
[169] S. Luo, Y. Zhao, and D. G. Truhlar, J. Phys. Chem. Lett. 3, 2975 (2012).

[170] L. Vega, J. Ruvireta, F. Viñes, and F. Illas, J. Chem. Theory Comput. 14, 395 (2018).

[171] L. Chiodo, L. A. Constantin, E. Fabiano, and F. Della Sala, Phys. Rev. Lett. 108, 126402 (2012).

[172] L. A. Constantin, L. Chiodo, E. Fabiano, I. Bodrenko, and F. D. Sala, Phys. Rev. B 84, 045126 (2011).

[173] J. M. Pitarke and A. G. Eguiluz, Phys. Rev. B 63, 045116 (2001).

[174] A. Patra, J. E. Bates, J. Sun, and J. P. Perdew, Proc. Natl. Acad. Sci. USA 114, E9188 (2017). 